\title{
Anesthesiologist and Critical Care
}

In 1940s, polio patients were supported with artificial ventilation by anesthetists. This can be considered as the beginning of critical care medicine. A radical transformation has been brought about in the practice of critical care over the last few decades-thanks to THE new drugs, procedures and equipment. Anesthesiologists in our country are increasingly involved in the management of ICUs.

Several factors have been identified to improve quality of care in an ICU. The first and foremost is the implementation of evidencebased practice. The second is the use of scoring systems to quantify the severity of illness. The third is the use of organ dysfunction scores. Last but not least, the development of guidelines and protocols improves patient outcome and reduces variability in management.

Several randomized controlled trials are currently evaluating cortisol replacement, tight glycemic control and activated protein C for reduction of mortality from sepsis. It is universally accepted that mechanical ventilation with low tidal volumes is preferable in patients with ARDS. Mortality from acute renal failure can be reduced with prompt use of dialysis. Similarly, liver transplant can reduce mortality from acute liver failure.

Complications of ICU management include ventilator-associated pneumonia (VAP), central venous catheter infections, and nosocomial infections. The measures recommended to prevent VAP are 30 degrees head high, subglottic aspiration of secretions and maintenance of gastric $\mathrm{pH}$ with sucralfate. Use of ultrasound, aseptic precautions and antibiotic-coated catheters can reduce catheter-related infections.

This issue attempts to present various aspects of critical care in a simple and lucid manner.

Editor-in-Chief Sunil Kumar Gvalani 